\title{
Medium range order and structural relaxation in As-Se network glasses through FSDP analysis
}

\author{
R. Golovchak ${ }^{\mathrm{a}, *}$, P. Lucas ${ }^{\mathrm{b}}$, J. Oelgoetz $^{\mathrm{a}}$, A. Kovalskiy ${ }^{\mathrm{a}}$, J. York-Winegar ${ }^{\mathrm{a}}$, \\ Ch. Saiyasombat ${ }^{\mathrm{c}}$, O. Shpotyuk ${ }^{\mathrm{d}}$, M. Feygenson ${ }^{\mathrm{e}}$, J. Neuefeind $^{\mathrm{e}}$, H. Jain ${ }^{\mathrm{c}}$ \\ ${ }^{a}$ Department of Physics and Astronomy, Austin Peay State University, Clarksville, TN 37044, USA \\ ${ }^{b}$ Department of Materials Science and Engineering, University of Arizona, Tucson, Arizona 85712 \\ ${ }^{c}$ Department of Materials Science and Engineering, Lehigh University, 5 East Packer Avenue, Bethlehem, PA \\ 18015-3195, USA \\ ${ }^{d}$ Institute of Physics, Jan Dlugosz University of Czestochowa, al. Armii Krajowej 13/15, Czestochowa, \\ 42200, Poland \\ ${ }^{e}$ Chemical and Engineering Materials Division, Spallation Neutron Source, Oak Ridge National \\ Laboratory, Oak Ridge, Tennessee 37831, USA
}

\begin{abstract}
Synchrotron X-ray diffraction and neutron scattering studies are performed on As-Se glasses in two states: as-prepared (rejuvenated) and aged for $\sim 23$ years. The first sharp diffraction peak (FSDP) obtained from the structure factor data as a function of composition and temperature indicates that the cooperative processes that are responsible for structural relaxation do not affect FSDP. The results are correlated with the composition dependence of the complex heat capacity of the glasses and concentration of different structural fragments in the glass network. The comparison of structural information shows that density fluctuations, which were thought previously to have a significant contribution to FSDP, have much smaller contribution than the cation-cation correlations, presence of ordered structural fragments or cage molecules.
\end{abstract}

Keywords: glasses; powder diffraction; neutron scattering and diffraction; glass transitions

\footnotetext{
* Corresponding author. Present address: Department of Physics and Astronomy, Austin Peay State University, Clarksville, TN-37044, USA.

E-mail address: holovchakr@apsu.edu (R. Golovchak). Tel.: 1-931-2216361
} 


\section{Introduction}

Correlation between the medium-range order (MRO) [1-7] and structural relaxation [8] in network glasses is one of the least studied and most controversial problems in solid state physics. According to S.R. Elliott [1], MRO is defined as a higher level of structural organization beyond the short-range order (SRO) in amorphous (glassy) materials, extending up to $\sim 20 \AA$ from a given atom. It is common to classify MRO into three categories [1-6]: the short-range MRO ( 3-5 $)$, which indicates mutual orientation of neighbored coordination polyhedra comprising the glass network; the intermediate-range MRO (5-10 $\AA$ ), which originates from triplet correlations between network forming units (correlations between pairs of dihedral angles, which is usually associated with clusters formation); and the long-range MRO ( 10-20 A), which is assumed to be determined by local dimensionality (1D, 2D or 3D) of glass network.

From this perspective, the chalcogenide glasses $(\mathrm{ChG})$ can be considered as unique model objects, where all of these types of MRO can be purposefully realized. Thus, for example, different kind of network-forming units (pyramids on the basis of three-fold coordinated $\mathrm{P}, \mathrm{As}, \mathrm{Sb}$ or $\mathrm{Bi}$; tetrahedra on the basis of four-fold coordinated $\mathrm{Si}, \mathrm{Ge}$ or $\mathrm{Ga}$ cations; polymeric chains on the basis of $\mathrm{S}, \mathrm{Se}$, Te anions) can result in MRO focusing on a short-range or intermediate-range scales; degree of dimensionality of the network can be easily modified by varying the composition of a glass (e.g. by increasing/decreasing the content of two-fold coordinated chalcogen atoms) leading to a controlled modification of long-range MRO, etc. $[9,10]$. In addition, high purity $\mathrm{ChG}$ can be obtained with SRO of relatively simple structural units that can be unambiguously derived from conventional structural probe data and verified by theoretical models, giving a possibility to link the observed regularities directly with the structural features $[11,12]$. Also, a very large database 
on various physical-chemical properties of these materials is available in scientific literature, which can be used as additional information for validating the interpretations of data [9-12].

Structural relaxation occurs as a result of thermodynamic forces driving the nonequilibrium glassy system towards a state of thermodynamic equilibrium of supercooled liquid [8]. The associated enthalpy losses $(\Delta H)$ can be measured with differential scanning calorimetry (DSC) by heating the glass through the glass-to-supercooled liquid transition [13]. Degree of structural relaxation depends on the waiting time below the glass transition temperature $\left(T_{g}\right)$, the relevant processes being known as physical ageing (excludes any chemical reactions, crystallization or phase separation) $[13,14]$. The greater ageing duration, the greater is the enthalpy loss $(\Delta H)$ expressed through the endothermic peak at $T_{g}$ in DSC scans [13]. In general, the $\Delta H$ follows a sigmoidal time dependence, which is defined by a choice of ageing temperature $T_{a}$ in respect to $T_{g}$ (increasing $T_{a}$ accelerates structural relaxation kinetics) [8]. For glasses with low $T_{g}$, significant enthalpy losses can be observed at room temperature over a short period of time, while for glasses with higher $T_{g}$, longer periods of storage are needed to observe physical ageing at room temperature [15-17]. The investigated arsenic selenide glasses show decrease in the value of physical ageing at room temperature towards the stoichiometric $\mathrm{As}_{2} \mathrm{Se}_{3}$ composition [17], which has the highest $T_{g}$ among all AsSe glasses $[9,11]$.

Different theoretical and experimental methods have been used so far to elucidate structural transformations during physical ageing in ChG. Most popular experimental techniques include Raman spectroscopy, nuclear magnetic resonance (NMR), extended X-ray absorption fine structure (EXAFS), high-resolution X-ray photoelectron spectroscopy (XPS) and positron annihilation lifetime spectroscopy (PALS) [14-18]. However, the data from these techniques show insignificant difference between the SRO structural parameters of the aged and non-aged glasses. The SRO remains practically invariant during physical aging. Although some minor structural transformations were detected, like $5-7 \%$ redistribution of Se atoms 
between different structural fragments [14-16], they could not explain the large changes in $T_{g}$ value and enthalpy losses $(\Delta H)$ observed with thermal analysis technique after prolong storage [17], as well as plateaus and steep regions in long-term kinetics of enthalpy relaxation [13]. Therefore, significant changes at MRO were anticipated. The deviations from monotonic linear temperature dependence of nearest neighbor distance and Debye-Waller factor found recently with quick EXAFS [19] had created additional reasoning for such expectations.

To study possible correlations between the MRO in $\mathrm{ChG}$ and their structural relaxation (physical ageing), the same as-prepared (rejuvenated) and aged samples should be investigated with the probes sensitive to the changes at MRO and those giving the magnitude of structural relaxation or physical ageing effect. However, contrary to structural relaxation processes, which can be conveniently characterized with thermal analysis techniques, the experimental investigation of MRO is a non-trivial task. Traditional structural probes such as X-ray, neutron or electron diffraction are sensitive to pair correlations between atoms, and therefore provide very limited information on MRO features. On the other hand, vibrational spectroscopies such as IR or Raman are more sensitive to the mutual correlations between coordination polyhedra, but usually they are suffered from the ambiguities in the interpretation of experimental spectra due to a great number of different possible vibrational modes that overlap. The information on MRO, which can be obtained using diffraction methods, is believed to be contained in the so-called first sharp diffraction peak (FSDP) [1-7]. It is observed as a prominent low- $Q\left(0.8<Q<1-1.6 \AA^{-1}\right)$ feature in the structure factor $S(Q)$ of network glasses. Although FSDP has been a subject of extended interest in the last three decades, its origin is still debated [20-25]. It is clear that FSDP can arise for various reasons in different systems [20,23,25], but in general it reflects features of the glass network at both the MRO and SRO length scales, for example, homopolar bonds, cage molecules, random/nonrandom distribution of the network-forming clusters, prevailing structural motif, etc. $[3,23,25]$. A characteristic length scale associated with the FSDP position typically 
implies 2-4 interatomic distances $(\sim 3.5-10 \AA)$, which correspond to the intermediate MRO. It was found earlier that the FSDP is distinguished from other $S(Q)$ peaks by at least two anomalies relative to the usual behavior of $S(Q)$ in solids: (i) it remains invariant with increasing temperature (sometimes may even become more intense and narrower) in contrast to the thermal broadening of usual peaks in $S(Q)$ [26-28], and (ii) its amplitude decreases with increasing pressure, again contrary to the response of other $S(Q)$ peaks $[29,30]$. The models used to explain the FSDP rely on the existence of layer-like structures in the glass network or regularities in the void distribution [1-5,31]. Others have recently proposed the FSDP as having a topological origin, arguing for a structural origin of the so-called topological "intermediate phase" in $\mathrm{As}_{\mathrm{x}} \mathrm{Se}_{1-\mathrm{x}}$ glasses [24]. The neutron diffraction with isotopic substitution and anomalous X-ray scattering clearly show that the main contribution to the FSDP in network glasses usually comes from network-forming cation-cation (metal-metal) correlations $[21,22]$, although the physical picture of these correlations in real glass structure remains unclear. A review of the theories of the FSDP for amorphous materials is given by Moss \& Price [31] and Wright [3].

To determine the potential of FSDP analysis in application to MRO and structural relaxation in network glasses, we have studied FSDP compositional trends in $\mathrm{As}_{x} \mathrm{Se}_{1-x}$ glasses $(0<x<0.55)$ with complementary X-ray diffraction (XRD) and neutron scattering techniques. Samples aged over a long period of time are compared to rejuvenated (thermal prehistory is erased by the annealing above the glass transition temperature $T_{g}$ ) samples derived from the same aged material, to investigate the influence of isothermal structural relaxation. Some of the aged Se-rich samples are additionally studied with neutron scattering in-situ during their heating through the glass transition interval, to study the effect of nonisothermal structural relaxation. The obtained evolution of FSDP parameters with composition and temperature is correlated with isothermal and non-isothermal structural relaxation data of temperature modulated DSC (TMDSC). 


\section{Experimental}

The samples of binary $\mathrm{As}_{x} \mathrm{Se}_{100-x}(x=10,20,30,40,45,50,53,55)$ were prepared in 1985 by conventional melt-quenching route in evacuated quartz ampoules from a mixture of high purity (99.999 \%) As and Se precursors. The furnace was rocked to obtain highly homogeneous melt. All ingots were quenched by switching off the furnace. Amorphous state of the as-prepared chalcogenide glasses was established visually by a characteristic conch-like fracture as well as by X-ray diffraction. All the samples were then sealed in hermetic plastic bags and stored in the dark, in sealed plastic bags under ambient conditions for $\sim 27$ years before the measurements. The purity and the composition of the samples after the storage were confirmed by X-ray photoelectron spectroscopy [32]. Before the experiments, part of the aged samples were rejuvenated in order to achieve the structural state close to the initial asprepared one $[9,12,13]$. This procedure comprised heating the samples above $T_{g}$, waiting equilibrium at $\left(T_{g}+30\right) \mathrm{K}$ and subsequent cooling in the chosen regime.

The neutron scattering experiments were performed at Nanoscale-Ordered Materials Diffractometer (NOMAD) at Spallation Neutron Source, Oak Ridge National Laboratory [33]. The bulk samples were measured in $2 \mathrm{~mm}$ quartz capillaries in an argon atmosphere to reduce scattering from the air. The temperature was controlled with non-liquid argon Cobra cryostream from Oxford Cryosystems. Three independent measurements for each sample's composition and thermal history were performed to confirm the reproducibility of the results. The high-energy XRD experiment was performed at the beamline 11-ID-B, Advanced Photon Source, Argonne National Laboratory, using X-rays with wavelength $0.2127 \AA$ and position sensitive Perkin Elmer amorphous silicon detector. These measurements were carried out at room temperature using coarse powdered (with mortar and pestle) samples sealed in Polyimide capillaries. 
In general, the scattered intensity $I(Q)$ from an array of atoms is given by three terms:

$$
I(Q)=I_{\text {self }}(Q)+I_{\text {int }}(Q)+I_{\text {inc }}(Q)
$$

where $Q=4 \pi \lambda \sin \theta$ is the scattering vector ( $2 \theta$ is the scattering angle and $\lambda$ is the $\mathrm{x}$-ray or neutron wavelength).

The first $I_{\text {self }}(Q)$ term in equation (1) is the sum of scattering from individual atoms; the second $I_{\text {int }}(Q)$ term represents interference scattering from different atoms and contains all the structural information in terms of spatial distribution of constituent atoms; and the third $I_{\text {inc }}(Q)$ term accounts for incoherent scattering. The first and third terms in equation (1) are usually subtracted from the experimentally measured $I(Q)$ using appropriate polarization, absorption and normalization corrections [6,33]. In the present experiment, neutron scattering data were normalized with respect to the scattering from solid vanadium rod, and the XRD data were corrected for background scattering, X-ray transmission and Compton scattering using PDFgetX2 software package [34]. After correction, the structure factor $S(Q)$, which contains information on the atomic pair correlations in the investigated material, was extracted from the $I(Q)$ data. For a glass or liquid with two atom types $\alpha$ and $\beta$ (e.g. As and Se in the case under consideration) [6]:

$$
S(Q)-1=\sum_{\alpha} \sum_{\beta} W_{\alpha \beta}\left(S_{\alpha \beta}(Q)-1\right)
$$

where the double summation covers the partial structure factors $S_{\alpha \beta}(Q)=\sin Q r_{\alpha \beta} / Q r_{\alpha \beta}$ between all pairs of atoms $\left(S_{\alpha \alpha}, S_{\beta \beta}\right.$ and $\left.S_{\alpha \beta}\right) ; r_{\alpha \beta}$ is the distance between atoms $\alpha$ and $\beta ; W_{\alpha \beta}$ are the normalized weights for the partial structure factors.

Temperature dependent in situ neutron scattering studies were performed on Se-rich aged samples at $2 \mathrm{~K} \cdot \mathrm{min}^{-1}$ heating rate. Heating was provided by hot argon gun with a thermocouple located just below the samples using Cobra cryostream temperature controller.

\section{Results}


Structure factors $S(Q)$ obtained from the neutron scattering and high-energy XRD experiments on the aged and rejuvenated $\mathrm{As}_{x} \mathrm{Se}_{100-x}$ glasses are shown in Fig. 1 for representative compositions. The FSDP is clearly visible for all the investigated Ascontaining glasses but not for pure Se, which is a typical behavior for the As-Se glass system [23]. Its characteristic parameters (position, width and height) are obtained using Gaussian fit of FSDP and the first principal peak (PP1) of $S(Q)$ spectrum after linear background subtraction and normalization to PP1 peak intensity (inserts to Fig. 1). After such a procedure, any observed difference between the overall principal peak intensities in $[S(Q)-1]$ as obtained from NS and XRD data of the aged and rejuvenated samples (Fig. 1) is eliminated from further consideration, restricting our analysis solely to the changes in FSDP parameters. It should be noted that neither Gaussian nor Lorentzian function reproduced the exact shape of FSDP peaks, but the former was significantly better for the less intense peaks and was therefore used systematically for all investigated glasses, similar to Ref. [23]. The obtained average values for the FSDP position and width (full width at half maximum) are plotted in Fig. 2 as a function of As concentration. The results obtained from neutron scattering and XRD agree fairly well with each other. Compositional dependence of FSDP position (Fig. 2a) fully corresponds to a general trend observed earlier for $\mathrm{As}_{x} \mathrm{Se}_{100-x}$ glasses [23]: increase in As concentration $(x)$ leads to a decrease in FSDP position. Four regions can be distinguished in the observed compositional dependence: $x<\sim 20$, where the FSDP position is almost independent of As concentration; $\sim 20-40$ at.\% of As content, where compositional dependence becomes steeper; $40-50$ at.\% of As with the strongest dependence on As concentration; and $x>\sim 50$, where the slope of characteristic parameters decreases again. Similar kind of FSDP position dependence on composition was obtained earlier for $\mathrm{As}_{x} \mathrm{Se}_{100-x}$ glass system too, even, though, the first region $(x<\sim 20)$ was not considered to be meaningful [23]. 
Compositional dependence of FSDP width (Fig. 2b) as obtained from Gaussian fit of FSDP peak demonstrates an unexpected behavior. Instead of the predictable extremum at $x=40$ at. $\%$, which corresponds to stoichiometric $\mathrm{As}_{40} \mathrm{Se}_{60}$ composition and rigidity percolation point where many physical properties show extrema in compositional dependences [9-11,35], a broad maximum is observed at around $x \approx 30$ at.\% of As (Fig. 2b). Remarkably, a similar behavior was observed recently in the compositional dependences of fragility, activation energy for viscous flow, enthalpy of structural relaxation and heat capacity throughout and above the glass transition temperature domain [36]. Although a tendency of an increase in the width of FSDP after long-term physical ageing can be assumed from the obtained neutron scattering data for the aged and ex situ rejuvenated samples (Fig. 2b), the noise level precludes a strong confidence in such a conclusion.

To obtain more precise information on the possible changes in FSDP parameters caused by long-term physical ageing and to eliminate possible inaccuracies associated with ex situ rejuvenation of the samples, neutron scattering spectra were collected in situ during heating and cooling of Se-rich glasses with $2 \mathrm{~K} / \mathrm{min}$ rate through their glass transition interval. Raw $S(Q)$ spectra obtained in a heating mode for $\mathrm{As}_{20} \mathrm{Se}_{80}$ glass are shown in Fig. 3a as an example. Typical $S(Q)$ data after linear background subtraction, which have been used for fitting, are compared in Fig. $3 \mathrm{~b}$ for $\mathrm{As}_{20} \mathrm{Se}_{80}$ glass sample before heating (at $300 \mathrm{~K}$ ), at maximum temperature $(500 \mathrm{~K})$ and after cooling back to $300 \mathrm{~K}$. The obvious effect of heating can be observed for the PP1 peak, while changes in FSDP parameters as a function of temperature are within the experimental accuracy.

\section{Discussion}

As for crystalline Bragg peaks, it can be assumed that FSDP in a glass is an evidence of some kind of periodicity in real space, which owing to the FSDP position should occur at 
MRO scale [3]. The much greater width in comparison to the peaks of crystalline materials indicates that the associated Fourier component decays after relatively few periods, which means that such periodicity is not global (like long-range ordering in crystalline materials). It is shown for several vitreous stoichiometric $\mathrm{ChG}$ compositions that the FSDP position is close to the Bragg reflection peaks from certain interlayer planes in corresponded crystalline structures [3]. Thus, for example, for vitreous $\mathrm{As}_{2} \mathrm{~S}_{3}$ or $\mathrm{As}_{2} \mathrm{Se}_{3}$ the FSDP position is close to 020 Bragg reflection peak in the monoclinic structures of crystalline counterparts $\left(\mathrm{As}_{2} \mathrm{~S}_{3}\right.$ and $\mathrm{As}_{2} \mathrm{Se}_{3}$ ) [37,38]; in vitreous $\mathrm{GeSe}_{2}$ and $\mathrm{GeS}_{2}$ the FSDP is found to be consistent with 002 Bragg reflection in high-temperature modifications of $\mathrm{GeSe}_{2}$ and $\mathrm{GeS}_{2}$ crystals $[39,40]$. This led to a conclusion that $\mathrm{ChG}$ should possess fragments of layer-like structures reminiscent of corresponding crystalline compounds [4,6]. However, as it was shown by Wright [3], formation of such layer-like structures does not necessarily require residual layers of crystalline counterparts, but could be inferred directly from Zachariasen's model of random glass network [41]. According to this approach, a few adjacent cages in Zachariasen network, flattened in the same direction, can approximate a set of layers and give rise to the Fourier component necessary for generating the FSDP even without an actual layered structure formed in its traditional meaning [3]. The Van-der-Waals forces determining the size of such cages are assumed to be the same as those between the equivalent planes in the corresponding crystalline phase and, thus, FSDP reflects the characteristic periodicity associated with this type of interaction. This is considered as a reason why FSDP position for ChG roughly coincided with the interlayer Bragg peak for the relevant polycrystalline powders [3].

On the basis of these arguments, Wright has suggested that there is no unambiguous evidence through FSDP for the existence of MRO in vitreous silica and other single component network glasses in excess of that is expected from Zachariasen network [3]. Although such an explanation looks attractive, it cannot fully account for the nonlinear compositional dependences of FSDP parameters (position and height) in different ChG 
systems [23]. The reason is that Zachariasen's network is not always a good approximation of ChG structure, especially for non-stoichiometric chalcogen-rich glasses, whose structure is more polymer-like. Therefore, the FSDP could reflect spatial ordering between 1D and 2D structural fragments bonded with Van-der-Waals forces. Likewise, for the cation (e.g. Si, As or Ge)-rich compositions, the structure may include well-established MRO structural motifs, cage-like molecules or deviations from fully polymerized network. Therefore, it was further suggested on the basis of FSDP studies in binary Se-based ChG that FSDP reflects multiple changes in the glass network on both SRO and MRO scales: random vs. nonrandom distribution of the network-forming cation structural units, homopolar cation-cation bond formation, network-forming vs. modifier-like behavior, the appearance of cage molecules, and the effect of local coordination of the atoms [23].

It is obvious from the obtained results (Fig. 1) that FSDP becomes more pronounced with the increase of As concentration in the samples, testifying previous conclusion on the origin of FSDP from cation-cation correlations (As metal plays role of cations in these ChG) [23]. Simultaneously, FSDP position shows a clear shift towards lower $Q$ values (Fig. 2). As the position of FSDP should reflect some repetitive characteristic distance $\left(R \approx 2 \pi / Q_{1}\right)$ between the structural entities in real space, the obtained results suggest that this distance increases from $\sim 4.4 \AA$ for Se-rich $\mathrm{ChG}$ to $\sim 6.0 \AA$ for As-rich ones. Owing to the typical SRO distances in As-Se glasses (bond lengths are within the range 2.35-2.43 $\AA$ ) [9-11,42], we can ascertain that correlations which contribute to FSDP should occur on a scale of 2-3 coordination spheres. Therefore, it would be useful to compare the observed compositional dependence in FSDP position with statistics of structural fragments in these glasses, which would include at least triplet correlations between atoms. The quantitative information of that sort can be obtained with high-resolution XPS analysis, which gives exact moieties of Se-SeSe, As-Se-Se, As-Se-As, Se-As $<(\mathrm{Se})_{2}$, As-As $<(\mathrm{Se})_{2}$ and Se-As $<(\mathrm{As})_{2}$ structural fragments in glass network [43]. It can be clearly seen (compare Fig. 2a and Fig. 4a) that FSDP position 
starts to decrease (or first change the slope in the compositional dependence) with the appearance of As-Se-As complexes in ChG structure (roughly for $x>20$ compositions). Within the $40<x<50$ composition range, the FSDP decrease becomes steeper and coincides with the range where the concentration of As-As $<(\mathrm{Se})_{2}$ complexes increases drastically (Fig. $4 \mathrm{~b}$ ). Further change in the slope of FSDP compositional dependence at $x>50$ corresponds to the range of the increase in concentration of Se-As $<(\mathrm{As})_{2}$ complexes and decrease of the former As-Se-As and As-As $<(\mathrm{Se})_{2}$ ones (Fig. 4). So, we may conclude that FSDP position is dominated by MRO correlations within the neighboring Se-As $<(\mathrm{As})_{2}$, As-Se-As and AsAs $<(\mathrm{Se})_{2}$ structural fragments. This result is consistent with the recent first principles molecular dynamic simulations, suggesting dominant input into FSDP from As-Se partial structural factor $S_{\mathrm{AsSe}}(k)$ [24]. However, the minimum in FSDP width of the partial structure factor $S_{\mathrm{AsSe}}(k)$ obtained by Bauchy et al. [24] at a composition near $30 \%$ of As, and used to justify the structural origin of a so-called "intermediate phase" [44], is not confirmed by the present experimental results. Moreover, a more careful examination of the FSDP width $\Delta k_{\mathrm{FSDP}}$ in Fig. 3 of their work [24] shows that it is virtually identical for compositions with $40 \%, 30 \%$ and $25 \%$ As and clearly larger for $20 \%$ and $35 \%$ of As (Fig. 5).

Our investigations reveal a maximum of $\Delta k_{\mathrm{FSDP}}$ in the vicinity of $x=20-30$ (Fig. 2b). As the width of FSDP is indicative of the coherence length, following the well-known Scherrer equation for microcrystals (connecting the width of a Bragg peak with the average size of microcrystals), it seems reasonable to associate this parameter with the existence of some kind of domains in the glass structure. Following these arguments, the origin of the domains could be associated with density fluctuations in the vitreous matrix, "frozen in" as the supercooled liquid cools into below- $T_{g}$ regime. A hypothesis on the origin of FSDP from the density fluctuations in glass structure was previously put forward in Ref. [45,46], but was not further developed. Although the existence of density fluctuations in glass networks can be verified by numerous experimental techniques, such as small angle X-ray scattering (SAXS) [6,47], small 
angle neutron scattering (SANS) $[3,47,48]$ or light scattering [49], their correlation with FSDP parameters is not well established. To verify the hypothesis, glasses with various degrees of density fluctuation but same chemical nature should be studied thoroughly; As-Se ChG series seems to be a perfect model system for this purpose.

Recently, we have shown that the width and asymmetry of the peak of the imaginary part of heat capacity, $C_{p}$ "' $(T)$, as obtained from TMDSC scans, contains information on length scale and magnitude of density fluctuations in As-Se glass matrix [50,51]. When heated through the glass transition during TMDSC scans, the low density domains achieve molecular mobility first, giving rise to the low temperature wing of the $C_{p}{ }^{\prime}{ }^{\prime}(T)$ curves, whereas at higher temperatures high density domains start to relax too, being responsible for the high-temperature wing. Following this interpretation, the width of $C_{p}{ }^{\prime \prime}(T)$ peak can be associated with density fluctuation distribution and the length-scales of the domains [50]. Any asymmetry of this peak would be caused by an interplay between the relaxation of low and high density domains in the glass structure (in the absence of other phenomena like network depolymerization or phase separation), serving as a measure of magnitude of density fluctuations [51]. So, it is useful to compare the results of TMDSC obtained for the same glasses with FSDP parameters in the aged and rejuvenated states. As can be seen from Fig. 6, the compositional dependence of asymmetry in $C_{p}{ }^{\prime \prime}(T)$ peak correlates well with $\Delta k_{\mathrm{FSDP}}$ for the aged ChG (Fig. 6a), while $C_{p}{ }^{\prime \prime}(T)$ peak width more or less resembles the compositional dependence of $\Delta k_{\mathrm{FSDP}}$ for the rejuvenated ChG with $x<50$ (Fig. 6b). So, there appears to be some contribution from density fluctuations to the width of FSDP: the higher degree of density fluctuations as derived from $C_{p}$ "' $(T)$ peak makes the FSDP peak broader.

The density fluctuations are shown to evolve with time during isothermal structural relaxation (physical ageing) of Se-rich glasses at below- $T_{g}$ temperatures [51]. These changes are envisioned as a sequence of repeated cycles of increase/decrease in density fluctuations, leading to a general shrinkage of glass network after prolonged storage [13]. Corresponding 
changes in the balance between fast and slow relaxation times at $T_{g}$ were recorded with TMDSC as changes in the asymmetry of $C_{p}{ }^{\prime \prime}{ }^{\prime}(T)$ peak (Fig. 6) [51]. Also, significant changes in the fluctuation density distribution can be assumed from the changes in the width of $C_{p}$ ", (T) peak as a result of prolonged physical ageing. Nevertheless, only a slight (within the experimental accuracy) decrease in $\Delta k_{\mathrm{FSDP}}$ for Se-rich rejuvenated samples is inferred from the neutron scattering data as a result of long-term physical ageing (Fig. 2b). The observed discrepancy in the compositional dependences of $C_{p}$ ") $(T)$ peak width and asymmetry, and $\Delta k_{\mathrm{FSDP}}$ for the aged (Fig. 6a) and rejuvenated (Fig. 6b) samples suggests that density fluctuations have only a minor effect on the FSDP width. The influence, however, cannot be ruled out completely, since the shoulder at $Q \sim 1.4 \AA^{-1}$ is observed in compositions with very low As content and even in amorphous Se (both aged and rejuvenated samples, Fig. 7), which does not contain As cations at all. Although generally FSDP is not expected in pure Se, we believe this shoulder develops into a peak when we introduce As into its structure (track the FSDP evolution in the samples with low As content in Fig. 1). So, we hypothesize that low- $Q$ shoulder at PP1 of amorphous Se originates from density fluctuations in vitreous matrix, having contribution also to FSDP in As-Se samples with low As content. Indeed, in homogeneous As-poor compositions $(x<10)$ the As atoms are separated by long Se chains and the cation-cation (metal-metal) correlations should occur at the distances much longer than those, determined from the position of FSDP (shoulder). With an increase in As concentration, the cation-cation correlations overrule FSDP behavior.

There are other factors that influence FSDP width, which should be considered: the chemical nature of network itself (distribution of various fragments), general ordering at MRO scale due to increase in the concentration of As atoms, and appearance of ordered structural units in the glass matrix such as cage-like molecules or other crystalline-like structures. Thus, for example, demixing of $\mathrm{As}_{4} \mathrm{Se}_{4}$ and $\mathrm{As}_{4} \mathrm{Se}_{3}$ cage molecules from the network of As-rich glasses $(x>40)$ after rejuvenation [51] would cause FSDP to become 
sharper $\left(\Delta k_{\mathrm{FSDP}}\right.$ smaller) at $x>40$. At the same time, density fluctuations as obtained from TMDSC are shown to increase drastically for the rejuvenated samples in this compositional range (Fig. 6b) [51,52]. Therefore, FSDP has a complex origin, which cannot be described explicitly by only one structural parameter (concentration of cations, degree of density fluctuations, order/disorder ratio, etc.).

The fact that FSDP parameters do not change significantly after prolonged physical ageing (or rejuvenation) (Fig. 2) suggests on a low sensitivity of FSDP to structural perturbations at MRO scale beyond the first two coordination spheres. This conclusion is also supported by in-situ neutron scattering experiments on heating of the aged Se-rich glasses through the glass-to-supercooled liquid transition interval, which show no FSDP signature of non-isothermal structural relaxation at $T_{g}$ (Fig. 3) where the strong endothermic peak indicating cooperative structural rearrangements is observed with TMDSC [17].

At the same time, several regularities are noticed with respect to the temperature dependence of FSDP and PP1 parameters. During the heating of Se-rich samples the position of FSDP shifts towards lower $Q$ values with increasing temperature (Fig. 8, $\mathrm{As}_{10} \mathrm{Se}_{90}$ and $\mathrm{As}_{20} \mathrm{Se}_{80}$ samples) and follows almost the same path back during cooling, retaining its original value at room temperature (Fig. 8, $\mathrm{As}_{20} \mathrm{Se}_{80}$ sample). This effect is not observed for the Asrich samples (Fig. 9), where the position of FSDP was found to be almost independent of temperature. Additionally, a decrease in the slope of temperature dependence of FSDP position can be noticed for the Se-rich samples in the above- $T_{g}$ regime (Fig. 8). On the other hand, FSDP width demonstrates much weaker temperature dependence for Se-rich samples than for the As-rich glasses (Figs. 8,9), decreasing slightly in both cases with temperature (FSDP becomes slightly sharper). The neutron scattering data for vitreous silica showed previously a temperature dependence behavior similar to that of the FSDP position and width for Se-rich samples [53]. However, the available temperature dependent studies of FSDP in ChG have been restricted either to stoichiometric compositions or to the FSDP intensity 
(height) analysis. Thus, the anomalous temperature dependence of the FSDP height (that increases when approached $T_{g}$ ) has been reported for several stoichiometric compositions (like $\mathrm{As}_{40} \mathrm{Se}_{60}, \mathrm{GeSe}_{2}$ ) and As-S glasses by means of XRD studies [26-28,54]. This behavior was accompanied by a decrease in the intensity (height) of all other SRO peaks in $S(Q)$, their broadening and shifting to lower $Q$ values [26,27]. We have observed similar trends in $S(Q)$ of SRO peaks in the As-Se ChG. In particular, PP1 position and height decrease, and PP1 peak width increases with increasing temperature (Fig. 8,9). However, anomalous increase in FSDP height was observed neither for Se-rich, nor for As-rich glasses. Moreover, FSDP height was found to decrease after $T_{g}$ in over-constrained $\mathrm{As}_{50} \mathrm{Se}_{50}$ glass (Fig. 9). This discrepancy with previous results obtained through XRD studies can be attributed to the influence of the neighboring PP1 peak width, which increases with temperature and overlaps with FSDP. The method to determine FSDP height used previously was based on the comparison of absolute $S(Q)$ intensity regardless of the contribution from such broadening $[26,27]$, whereas we have determined this value through the fit of both FSDP and PP1 peaks after appropriate background subtraction. Perhaps, the physical origin of the information obtained through the neutron scattering and X-ray diffraction should be taken into account additionally. In fact, more in-situ studies on glasses of different families are needed to derive a general conclusion on the behavior of FSDP with temperature.

\section{Conclusions}

The neutron scattering and synchrotron XRD studies of As-Se glass series shows that structural relaxation during long-term natural storage has little impact on FSDP parameters, which are much more sensitive to the glass chemical composition variation. Therefore, it is concluded that the FSDP for this system is more sensitive to the regularities in SRO and MRO on a scale within the first two coordination spheres. Consequently, FSDP analysis is not 
suitable for the investigation of structural relaxation in network glasses, which is essentially a cooperative process. Absence of the pronounced peculiarities in temperature dependence of FSDP parameters through the glass transition interval, where a strong endothermic peak is usually observed for the aged samples by thermal analysis, just confirms this conclusion. By comparing the compositional dependence of the width and asymmetry of out-of phase component, $\Delta C_{P}$ "' $(T)$, of the complex heat capacity $C^{*}(T)$ at $T_{g}$ and compositional dependence of FSDP parameters, it is shown that density fluctuations, which were thought previously to have a significant contribution to FSDP, have smaller impact on FSDP than the cation-cation correlations.

The width and height of FSDP during heating/cooling do not show any strong dependence on the temperature. Thus, anomalous increase in the intensity of FSDP with temperature claimed earlier is not observed. On the other hand, FSDP position for Se-rich samples exhibits a low-Q shift with temperature. The observed temperature dependence can be approximated by two linear curves with different slopes before and after $T_{g}$.

\section{Acknowledgments}

R. Golovchak acknowledges support from U.S. National Science Foundation (NSF Grant No. DMR-0844014), for his Research Exchange visit to Lehigh University through International Materials Institute for New Functionality in Glass (IMI-NFG). A. Kovalskiy admits funding from NSF's DMR-1409160 project. A portion of this research at Oak Ridge National Laboratory's Spallation Neutron Source was sponsored by the Scientific User Facilities Division, Office of Basic Energy Sciences, U.S. Department of Energy. Use of the Advanced

Photon Source was supported by the U.S. Department of Energy, Office of Science, Office of Basic Energy Sciences, under Contract No. DE-AC02-06CH11357. 


\section{References}

[1] S.R. Elliott, J. Non-Cryst. Solids 97\&98 (1987) 159.

[2] S.R. Elliott, Phys. Rev. Lett. 67, 6 (1991) 711.

[3] A.C. Wright, Phys. Chem. Glasses: Eur. J. Glass Sci. Technol. B 49, 3 (2008) 103.

[4] M. Popescu, Medium range order in chalcogenide glasses. In: Physics and application of non-crystalline semiconductors, ed. A. Andriesh and M. Bertolotti, Kluwer Academic Publishers, 1997.

[5] S.R. Elliott, J. Non-Cryst. Solids 182 (1995) 40.

[6] G. N. Greaves, S. Sen, Advances in Physics 56, 1 (2007) 1.

[7] G. Lucovsky, J.C. Phillips, Nanoscale Res. Lett. 5 (2010) 550.

[8] K.L. Ngai, Relaxation and diffusion in complex systems, Springer, New YorkDordrecht-Heidelberg-London, 2011.

[9] A. Feltz, Amorphous Inorganic Materials and Glasses, VCH, Weinheim, 1993.

[10] M. Popescu, Non-crystalline chalcogenides, New York, Boston, Dordrecht, London, Moscow, Kluwer Academic Publishers, 2002.

[11] Z.U. Borisova, Chalcogenide semiconducting glasses, Leningrad, Nauka, 1983.

[12] Chalcogenide Glasses: Preparation, properties and application, ed. J-L. Adam and X. Zhang, Woodhead Publishing series in Electronic and Optical Materials No.44, 2014.

[13] R. Golovchak, A. Kozdras, V. Balitska, O. Shpotyuk, J. Physics: Cond. Matter 24 (2012) 505106(10).

[14] R. Golovchak, O. Shpotyuk, A. Kozdras, B. Bureau, M. Vlcek, A. Ganjoo , H. Jain, Phil. Mag. 87 (2007) 4323.

[15] R. Golovchak, O. Shpotyuk, A. Kozdras, M. Vlcek, B. Bureau, A. Kovalskiy , H. Jain, J. Physics: Cond. Matter 20 (2008) 245101(7). 
[16] R. Golovchak, B. Bureau, O. Shpotyuk, V. Boyko , M. Hyla, J. Non-Cryst. Solids 377 (2013) 43.

[17] R. Golovchak, H. Jain, O. Shpotyuk, A. Kozdras, A. Saiter , J.M. Saiter, Phys. Rev. B 78 (2008) 014202(6).

[18] R. Golovchak, A. Ingram, S. Kozyukhin , O. Shpotyuk, J. Non-Cryst. Solids 377 (2013) 49-53.

[19] R. Golovchak, A. Kovalskiy, O. Shpotyuk , H. Jain, Appl. Phys. Letters 98 (2011) 171905.

[20] A. C. Wright, R. N. Sinclair, A. J. Leadbetter, J. Non-Cryst. Solids 71 (1985) 295.

[21] M. Arai, R. W. Johnson, D. L. Price, S. Susman, M. Gay, J. E. Enderby, J. Non-Cryst. Solids 83 (1986) 80.

[22] I. Petri, P. S. Salmon , H. E. Fischer, Phys. Rev. Lett. 84 (2000) 2413.

[23] E. Bychkov, C.J. Benmore , D.L. Price, Phys. Rev. B 72 (2005) 172107.

[24] M. Bauchy, M. Micoulaut, M. Boero , C. Massobrio, Phys. Rev. Let. 110 (2013) 165501.

[25] A. Bytchkov, M. Miloshova, E. Bychkov, Sh. Kohara, L. Hennet, D.L. Price, Phys. Rev. B 83 (2011) 144201.

[26] L.E. Busse , S.R. Nagel, Phys. Rev. Letters 47 (1981) 1848.

[27] L.E. Busse, Phys. Rev. B 29 (1984) 3639.

[28] S. Susman, K. J. Volin, D. G. Montague , D.L. Price, J. Non-Cryst. Solids 125 (1990) 168.

[29] H. Tsutsu, K. Tamura , H. Endo, Solid State Commun. 52 (1984) 877.

[30] M. Guthrie, C. A. Tulk, C. J. Benmore, J. Xu, J. L. Yarger, H.-K. Mao , R. J. Hemley, Phys. Rev. Lett. 93 (2004) 115502. 
[31] S.C. Moss , D.L. Price, Random Packing of Structural Units and the First Sharp Diffraction Peak in Glasses. In: Physics of Disordered Materials, ed. D. Adler, H. Fritzsche \& S. R. Ovshinsky, Plenum, New York, 1985.

[32] R. Golovchak, A. Kovalskiy, A.C. Miller, H. Jain , O. Shpotyuk, Phys. Rev. B 76 (2007) 125208.

[33] J. Neuefeind, M. Feygenson, J. Carruth, R. Hoffmann , K. Chipley, Nuclear Instruments and Methods B 287 (2012) 68.

[34] X. Qiu, J.W. Thompson, S. J. L. Billinge, J. Appl. Crystallogr. 678 (2004) 37.

[35] G. Yang, B. Bureau, T. Rouxel, Y. Gueguen, O. Gulbiten, C. Roiland, E. Soignard, J. L. Yarger, J. Troles, J.-Ch. Sangleboeuf, P. Lucas, Phys. Rev. B 82 (2010) 195206.

[36] G. Yang, O. Gulbiten, Y. Gueguen, B. Bureau, J.-Ch. Sangleboeuf, C. Roiland, E.A. King, P. Lucas, Phys. Rev. B 85 (2012) 144107.

[37] D. J. E. Mullen, W. Nowacki, Zeitschrift fiir Kristallographie, Bd. 136 (1972) 48.

[38] A.L. Renninger, B.L. Averbach, Acta Cryst. B 29 (1973) 1583.

[39] G. Dittmar, H. Schafer, Acta Crystallogr. B 32 (1976) 2726.

[40] G. Dittmar, H. Schafer, Acta Crystallogr. B 31 (1975) 2060.

[41] W.H. Zachariasen, J. Am. Chem. Soc. 54 (1932) 3841.

[42] Y. Iwadate, T. Hattori, S. Nishiyama, K. Fukushima, Y. Mochizuki, M. Misawa , T. Fukunaga, J. Phys. \& Chem. Solids 60 (1999) 1447.

[43] R. Golovchak, A. Kovalskiy, O. Shpotyuk , H. Jain, Solid State Commun. 165 (2013) 22.

[44] P. Boolchand, P. Chen, D. I. Novita, B. Goodman, in: Rigidity and Boolchand Intermediate Phases in Nanomaterials, ed. M. Micoulaut, M. Popescu, INOE, Bucharest, 2009.

[45] J. Swenson, L. Borjesson, R.L. McGreevy, W. S. Howells, Phys. Rev. B 55, 11 (1997) 236. 
[46] P.H. Gaskell , D.J. Wallis, Phys. Rev. Letters 76, 1 (1996) 66.

[47] A.C. Wright, R.A. Hulme , R.N. Sinclair, Phys. Chem. Glasses 46 (2005) 59.

[48] E.A. Porai-Koshits, Ann. Rev. Mater. Sci. 6 (1976) 389.

[49] K. Takahara, H. Saito , T. Inoue, Polymer 40 (1999) 3729.

[50] E. Donth, The glass transition Relaxation dynamics in liquids and disordered materials. Springer, Materials Science, Berlin, 2001.

[51] R. Golovchak, J. Oelgoetz, M. Vlcek, A. Esposito, A. Saiter, J.-M. Saiter, H. Jain, J. Chem. Physics 140 (2014) 054505.

[52] A. Saiter, J.-M. Saiter, R. Golovchak, M. Shpotyuk, O. Shpotyuk, J. Physics: Cond. Matter 21 (2009) 075105(7).

[53] S. Susman, K.J. Volin, D.G. Montague , D.L. Price, Phys. Rev. B 43 (1991) 11076.

[54] S. Susman, D.L. Price, K.J. Volin, R.J. Dejus , D.G. Montague, J. Non-Cryst. Solids $106(1988) 26$. 


\section{Figure captions}

Fig. 1. Structure factors $S(Q)$ as obtained from neutron scattering (NS) and high-energy XRD data for aged and rejuvenated $\mathrm{As}_{\mathrm{x}} \mathrm{Se}_{100-\mathrm{x}}$ glasses. Insets show the magnified region with FSDP and PP1 peaks, normalized to PP1 intensity after background subtraction.

Fig. 2. Compositional dependence of FSDP position (a) and width (b) for aged and rejuvenated $\mathrm{As}_{\mathrm{x}} \mathrm{Se}_{100-\mathrm{x}}$ glasses as obtained from fitting.

Fig. 3. Temperature evolution of NS data obtained in situ during heating of aged $\mathrm{As}_{20} \mathrm{Se}_{80}$ sample (a); comparison of the FSDP and PP1 peaks after baseline subtraction for sample at different temperatures and thermal prehistory (b).

Fig. 4. Compositional dependences of structural moieties for Se-based (a) and As-based (b) complexes as obtained from XPS analysis [43].

Fig. 5. Comparison of the FSDP data presented in Ref. [24] for the partial structure factor $S_{A s S e}(k)$. FSDP peak is distinguished by dashed rectangle.

Fig. 6. Comparison of compositional dependences of FSDP width (circles), $C_{p}{ }^{\prime \prime}(T)$ peak width (triangles) and $C_{p}{ }^{\prime \prime}(T)$ asymmetry (asterisk) as obtained from TMDSC scans for aged (a) and rejuvenated (b) samples [51,52].

Fig. 7. Neutron scattering structure factors $S(Q)$ for aged and rejuvenated Se samples.

Fig. 8. Temperature dependences of FSDP and PP1 fit parameters for Se-rich $\mathrm{As}_{10} \mathrm{Se}_{90}$ and $\mathrm{As}_{20} \mathrm{Se}_{80}$ samples (open symbols - during heating, closed symbols - during cooling. Lines correspond to linear fits (heating mode), which slopes are indicated on graphs. 
Fig. 9. Temperature dependences (heating mode) of FSDP and PP1 fit parameters for As-rich $\mathrm{As}_{50} \mathrm{Se}_{50}$ example. Lines correspond to linear fits, which slope is indicated on a graph.
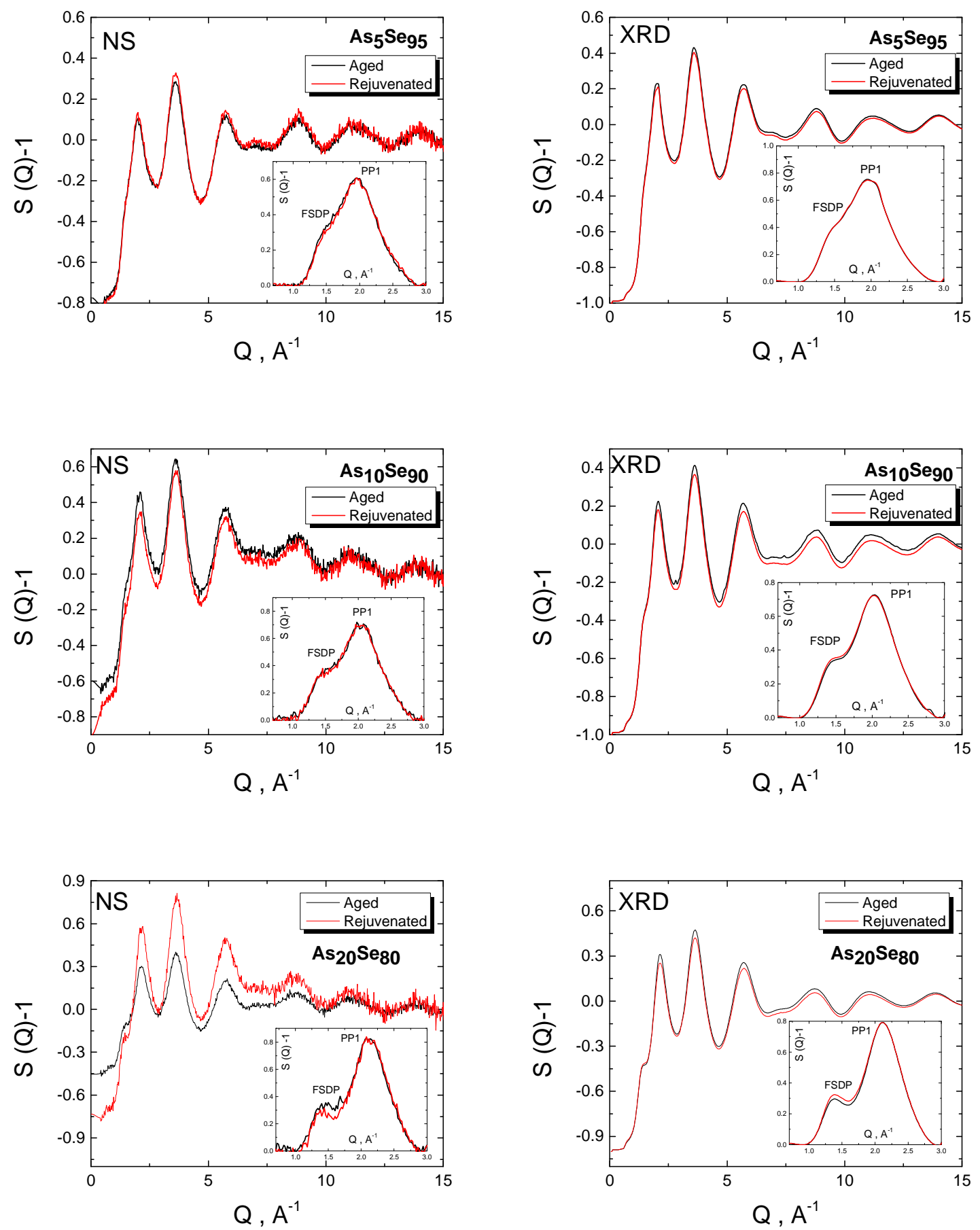

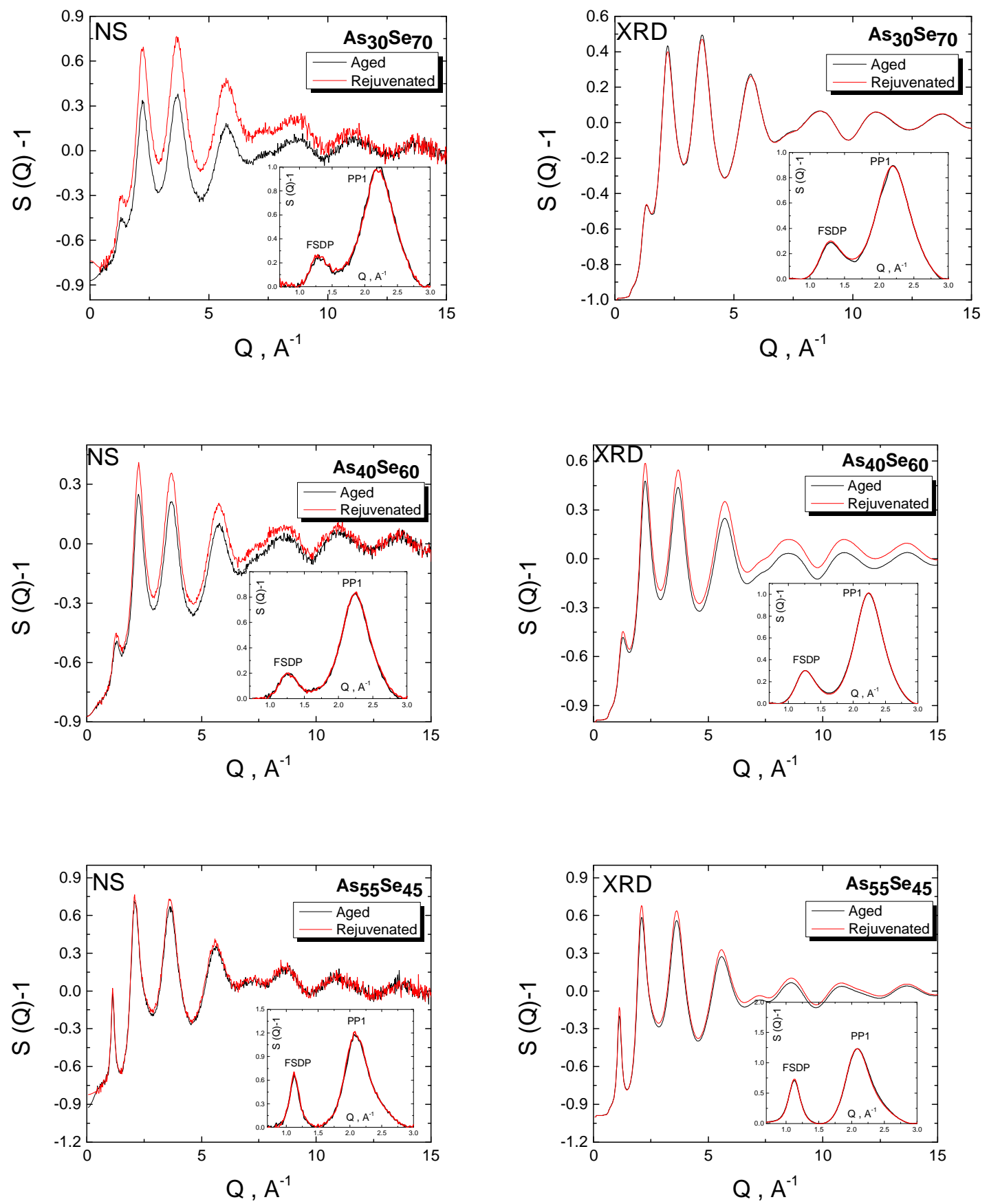

Figure 1. 


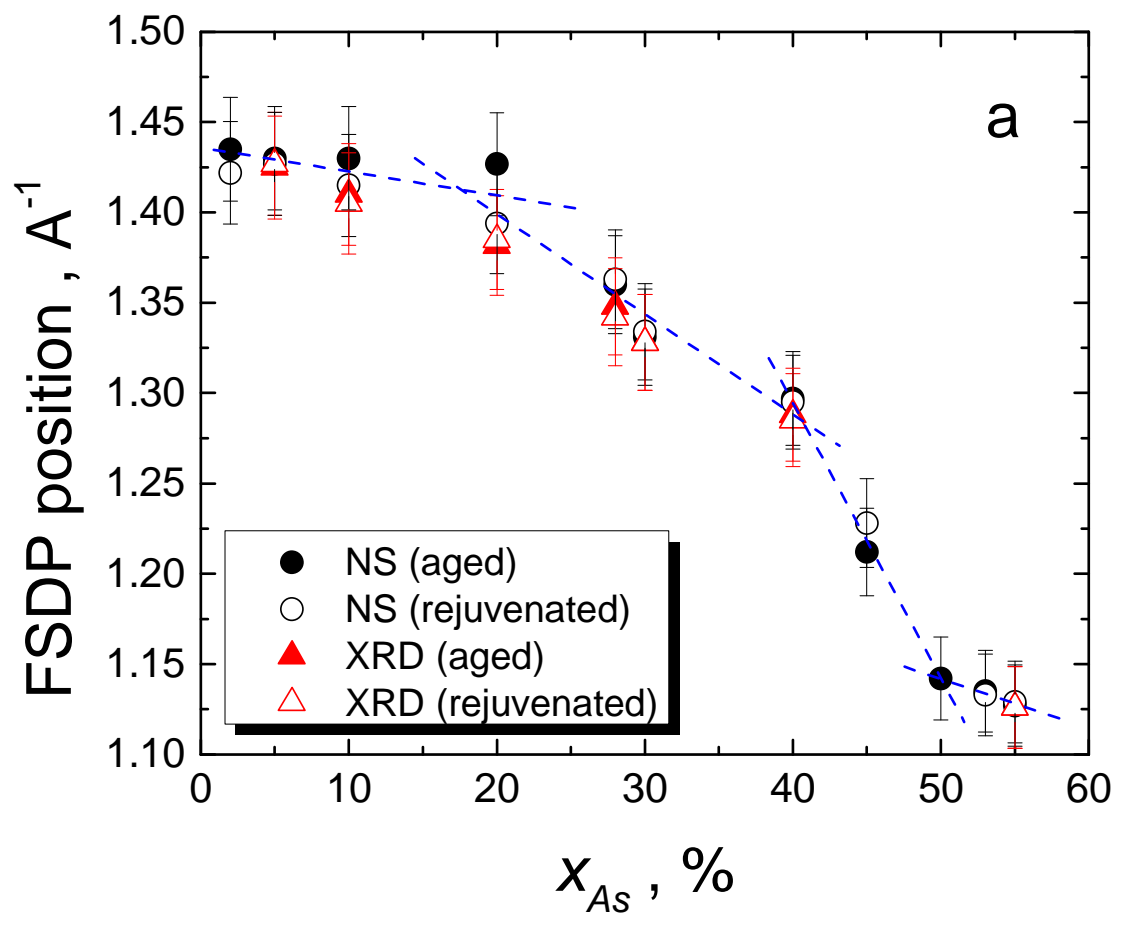




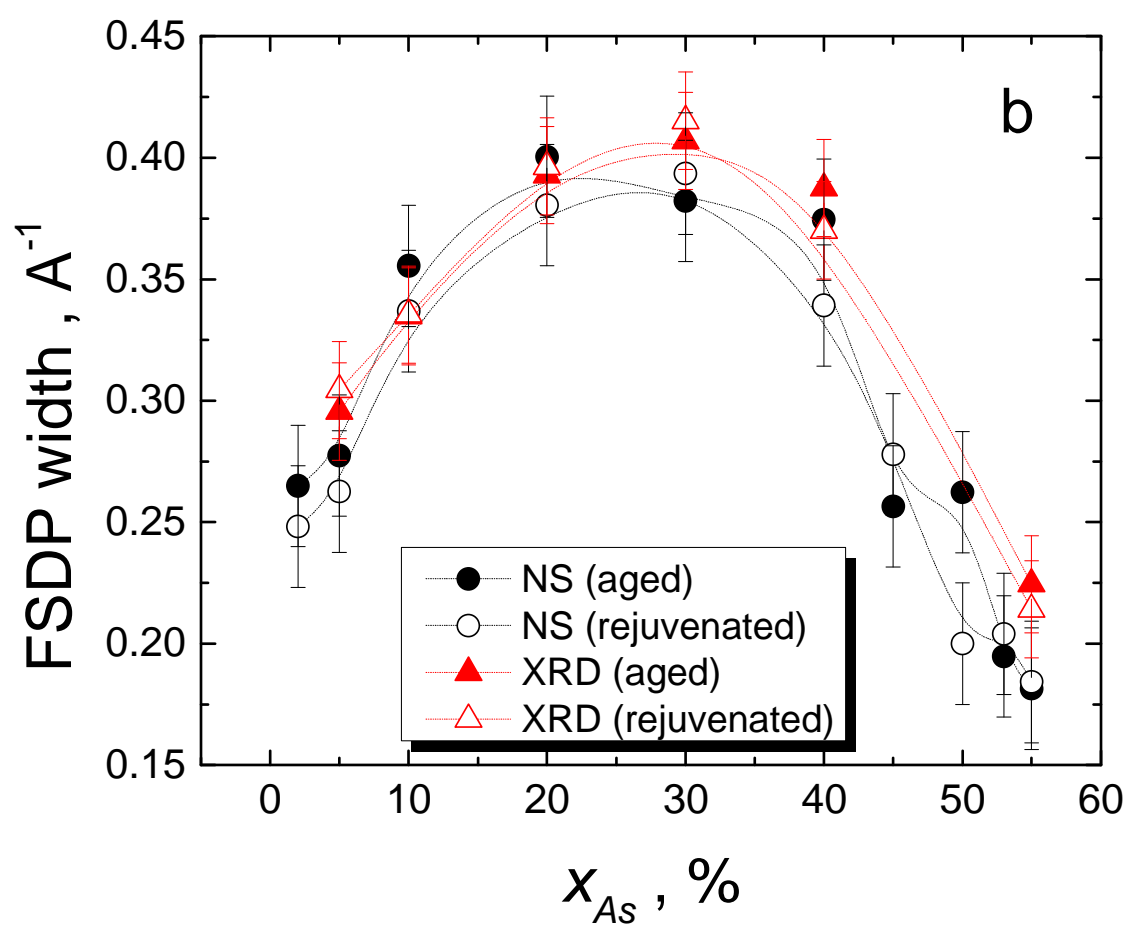

Figure 2.

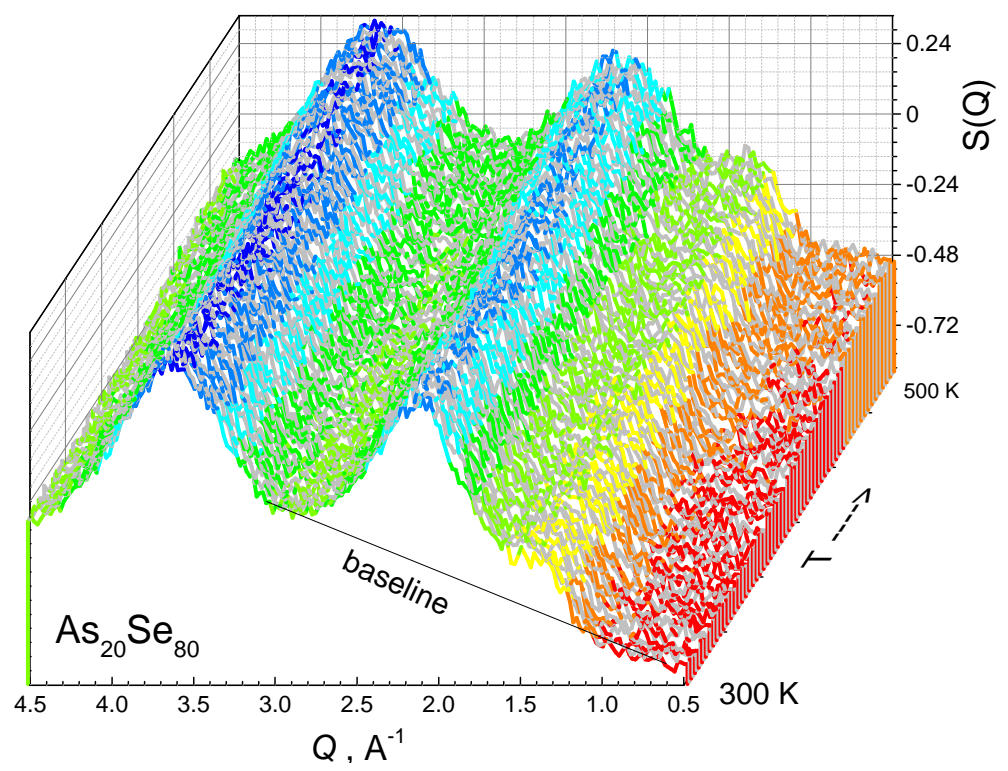

(a) 


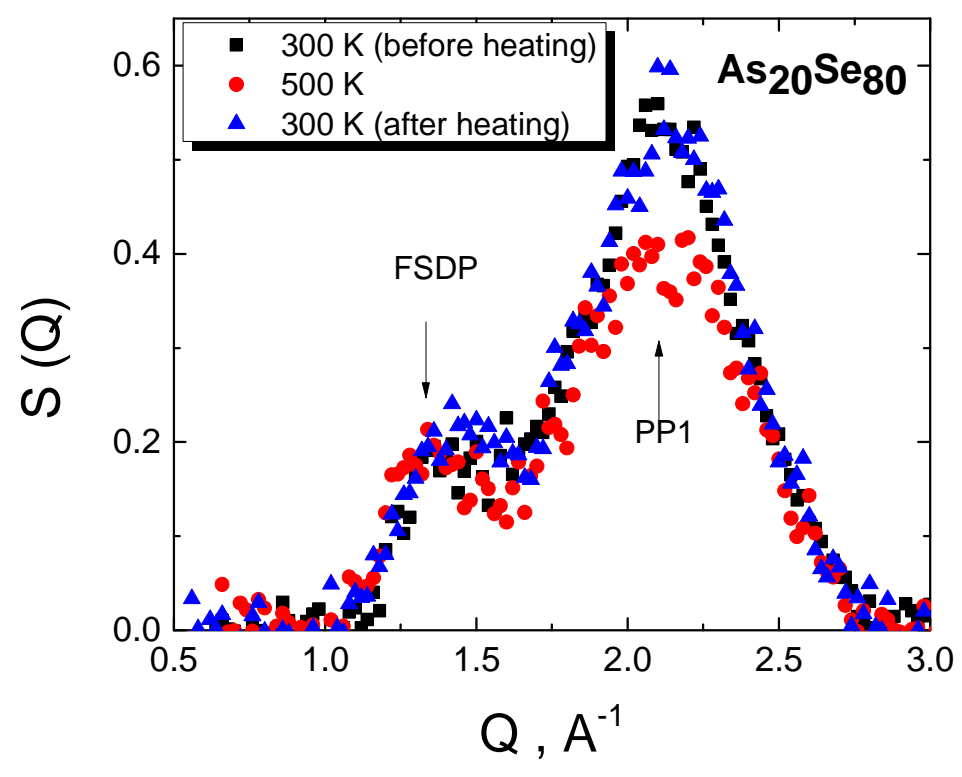

Figure 3. 

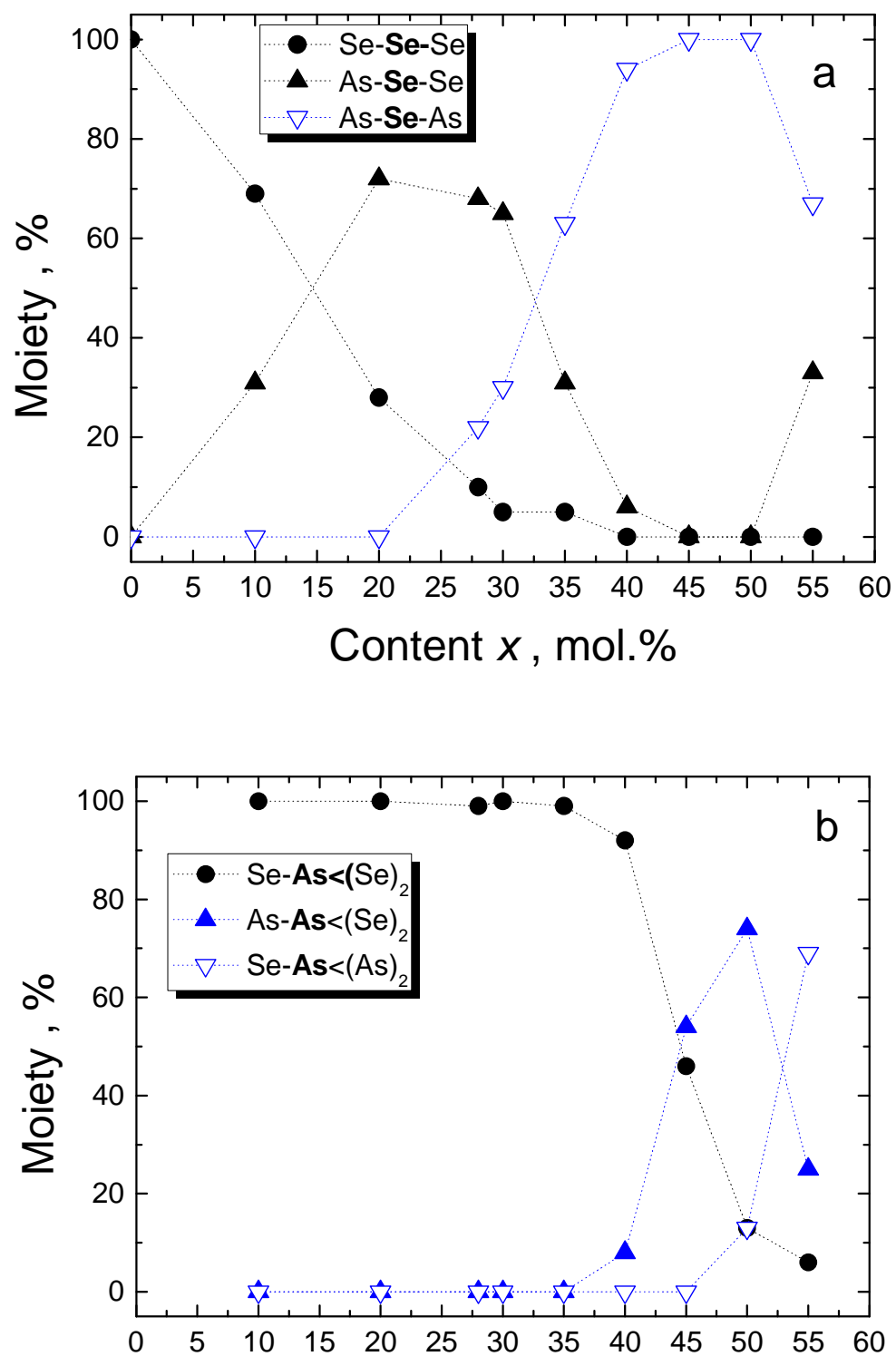

Content $x$, mol.\%

Figure 4. 


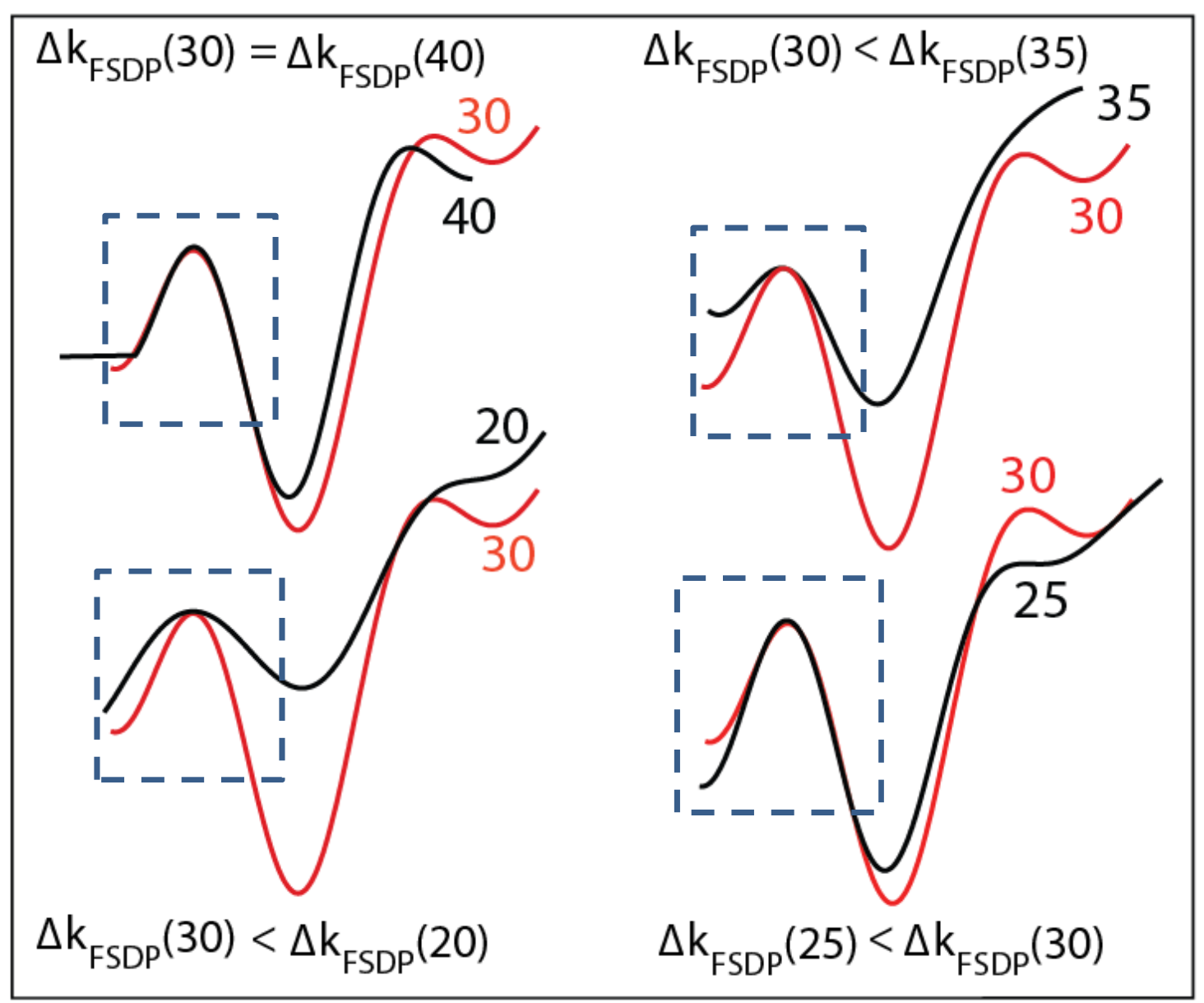

Figure 5. 

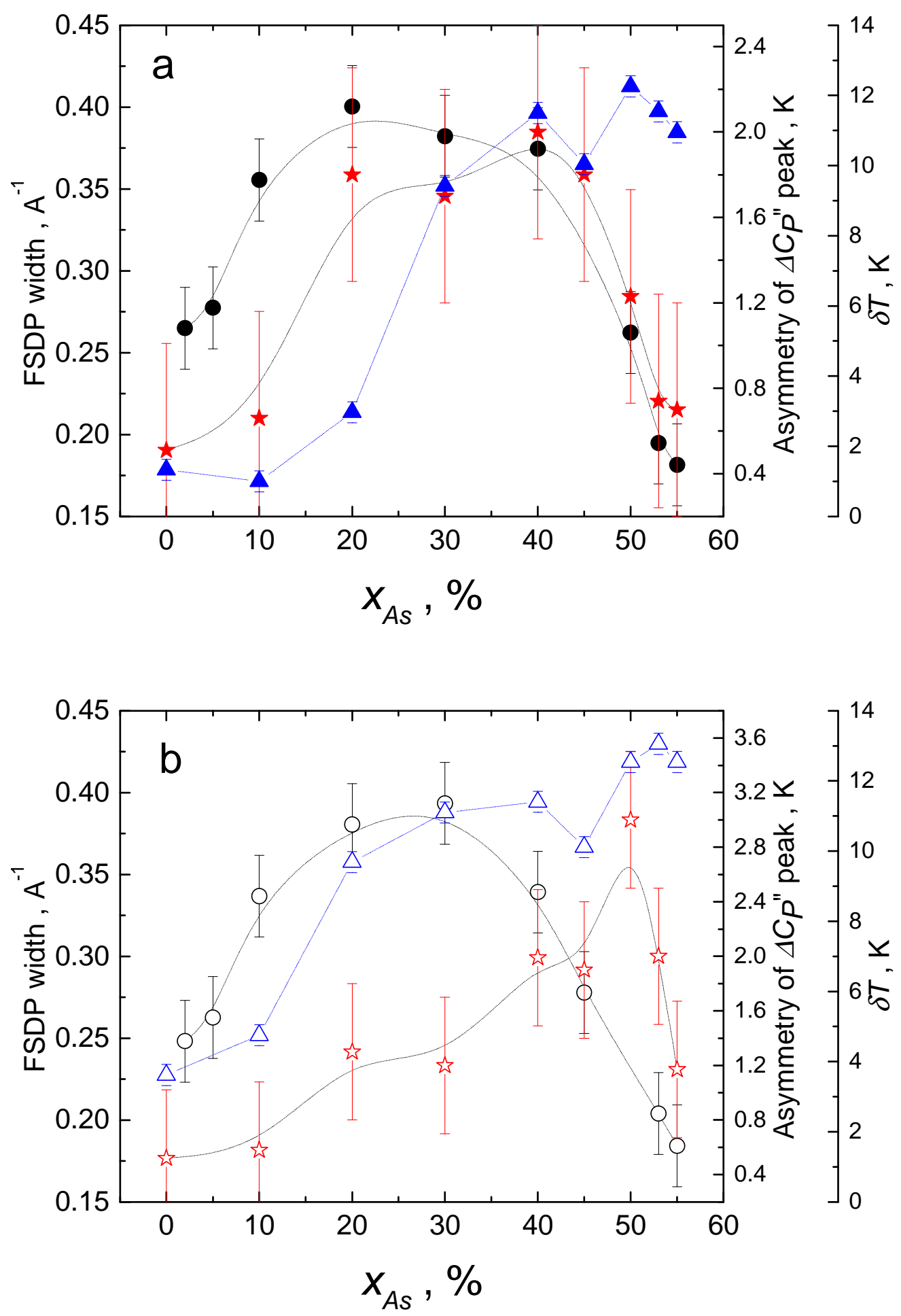

Figure 6. 

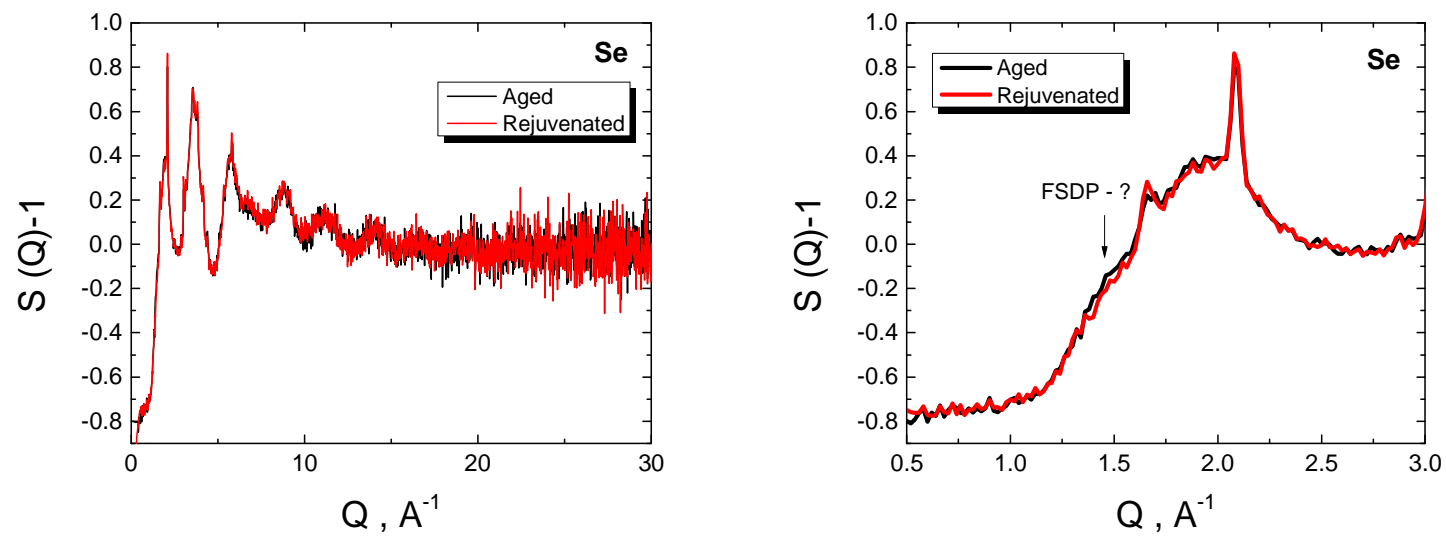

Figure 7. 

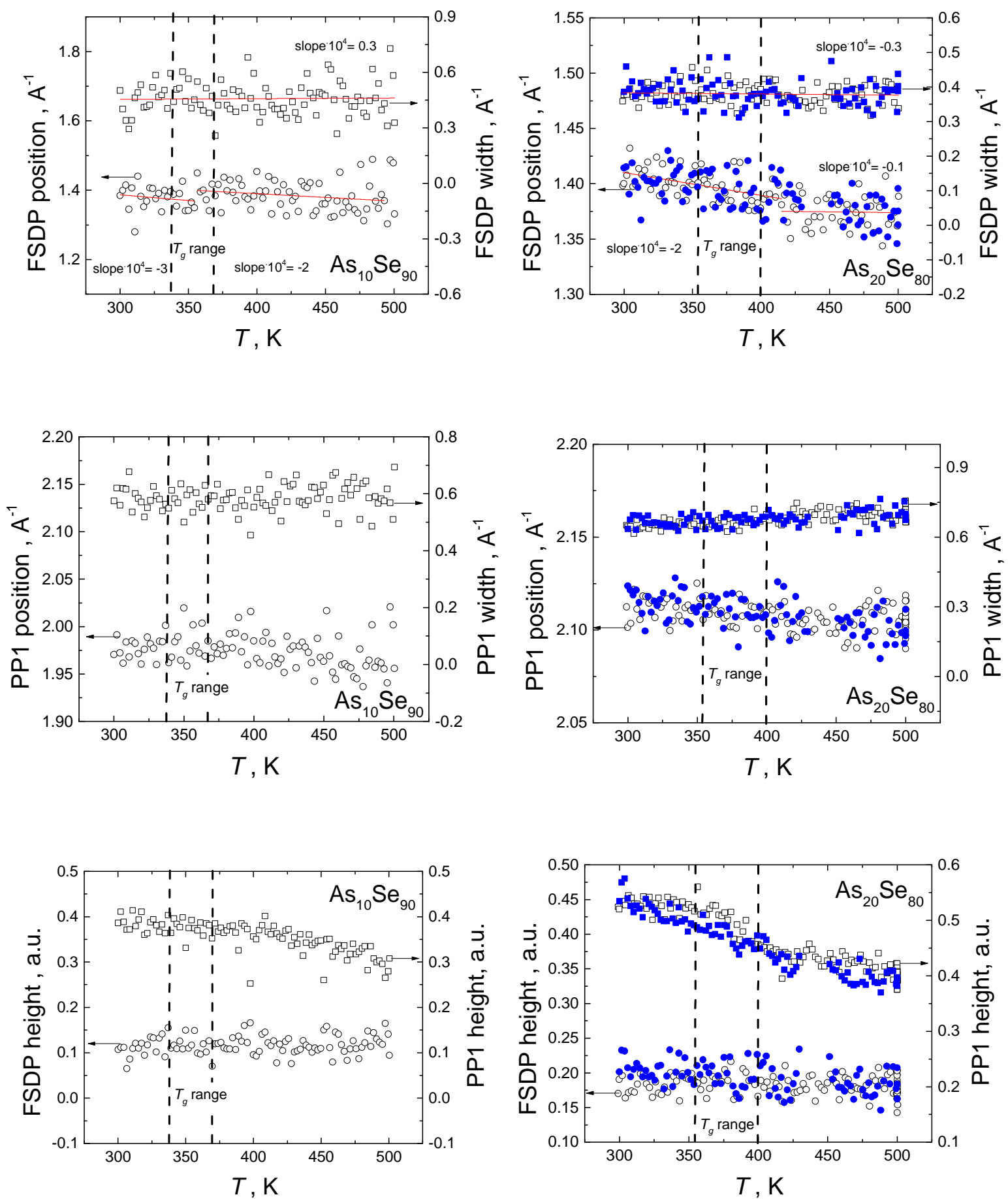

Figure 8. 

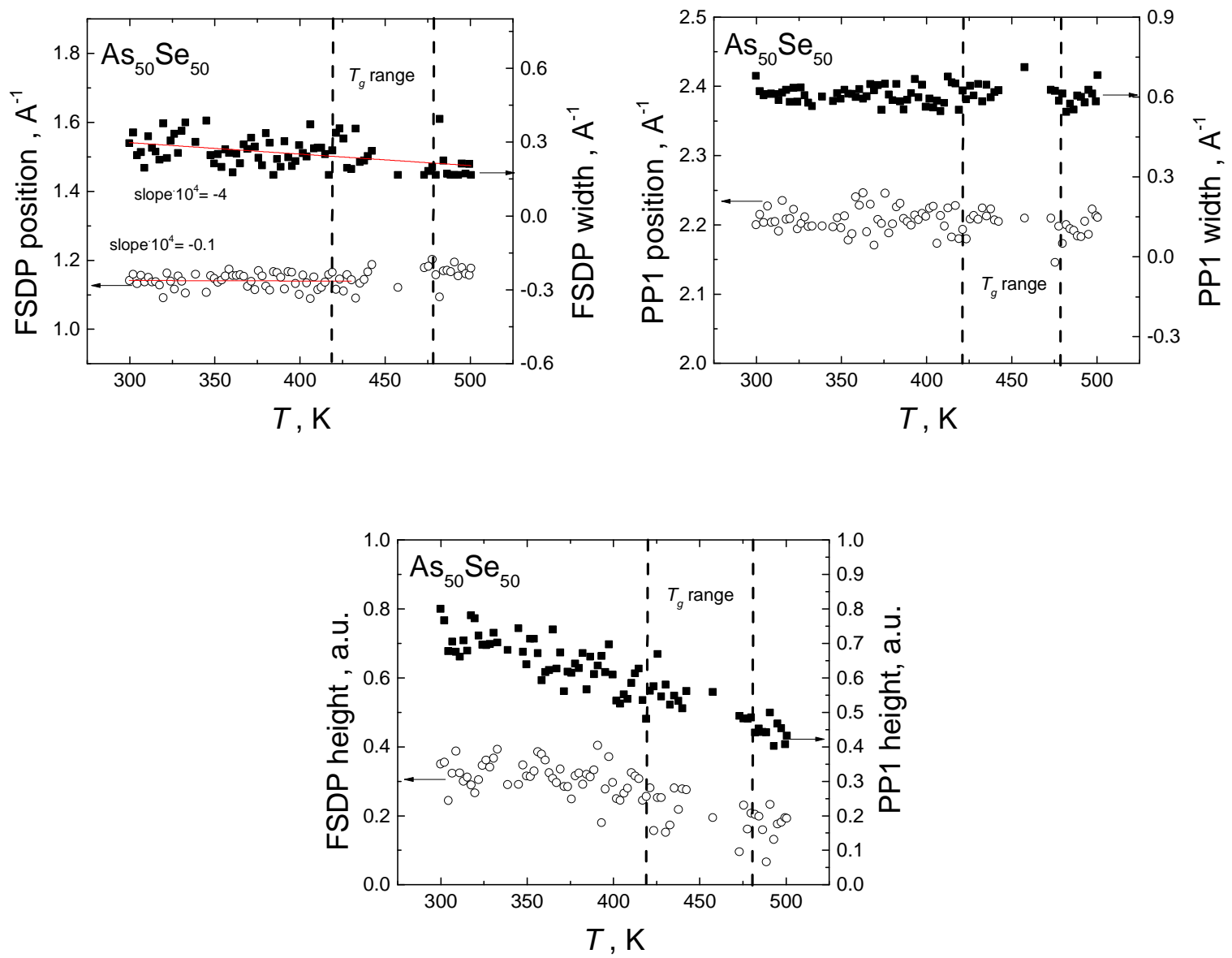

Figure 9. 